\title{
Long non-coding RNA HOTAIR promotes glioblastoma cell cycle progression in an EZH2 dependent manner
}

\author{
Kailiang Zhang ${ }^{1,2, *}$, Xiaotian Sun $2,3,{ }^{*}$, Xuan Zhou ${ }^{4}$, Lei Han ${ }^{1}$, Luyue Chen ${ }^{1}$, Zhendong \\ Shi $^{1}$, Anling Zhang ${ }^{1}$, Minhua Ye $^{5}$, Qixue Wang ${ }^{1}$, Chaoyong Liu ${ }^{6}$, Jianwei Wei ${ }^{1}$, Yu \\ Ren ${ }^{7}$, Jingxuan Yang ${ }^{2}$, Jianning Zhang ${ }^{1}$, Peiyu $\mathrm{Pu}^{1}$, Min $\mathrm{Li}^{2}$ and Chunsheng Kang ${ }^{1}$ \\ ${ }^{1}$ Department of Neurosurgery, Tianjin Medical University General Hospital; Laboratory of Neuro-Oncology, Tianjin Neurological \\ Institute; Key Laboratory of Post-trauma Neuro-repair and Regeneration in Central Nervous System, Ministry of Education; \\ Tianjin Key Laboratory of Injuries, Variations and Regeneration of Nervous System, Tianjin, China \\ ${ }^{2}$ Department of Medicine, Department of Surgery, The University of Oklahoma Health Sciences Center, Oklahoma City, OK, \\ USA \\ ${ }^{3}$ Department of Gastroenterology, Changhai Hospital, Second Military Medical University, Shanghai, China \\ 4 The Department of Otorhinolaryngology and Maxillofacial Oncology, Tianjin Medical University Cancer Institute and \\ Hospital; Key Laboratory of Cancer Prevention and Therapy, Tianjin Cancer Institute; National Clinical Research Center of \\ Cancer, Tianjin, China \\ ${ }^{5}$ Department of Neurosurgery, Second Affiliated Hospital of Nanchang University, Nanchang, Jiangxi Province, China \\ ${ }^{6}$ School of Materials Science and Engineering, Tianjin University, Tianjin, China \\ ${ }^{7}$ Tianjin Research Center of Basic Medical Science, Tianjin Medical University, Tianjin, China \\ * These authors contributed equally to this work \\ Correspondence to: Min Li, email: Min-Li@ouhsc.edu
}

Chunsheng Kang, email: kang97061@gmail.com

Keywords: Long non-coding RNA; HOTAIR; GBM; Cell cycle; EZH2

Received: August 27, $2014 \quad$ Accepted: November 04, $2014 \quad$ Published: November 04, 2014

This is an open-access article distributed under the terms of the Creative Commons Attribution License, which permits unrestricted use, distribution, and reproduction in any medium, provided the original author and source are credited.

\section{ABSTRACT}

The long non-coding RNA Hox transcript antisense intergenic RNA (HOTAIR) was recently implicated in breast cancer metastasis and is predictive of poor prognosis in colorectal and pancreatic cancers. We recently discovered that HOTAIR is a cell cycle-related IncRNA in human glioma, and its expression is closely associated with glioma staging and poor prognosis. Although lysine specific demethylase 1 (LSD1) and polycomb repressive complex 2 (PRC2) have been demonstrated to be functional targets of HOTAIR, how HOTAIR regulates glioma cell cycle progression remains largely unknown. In this study, we found that EZH2 (predominant PRC2 complex component) inhibition blocked cell cycle progression in glioma cells, consistent with the effects elicited by HOTAIR siRNA. However, the inhibition of LSD1 did not affect cell cycle progression in glioma cells. These results suggest that HOTAIR might regulate cell cycle progression through EZH2. Our intracranial mice model also revealed delayed tumor growth in HOTAIR siRNA- and EZH2 inhibitor-treated groups. Moreover, in HOTAIR knock-down cell lines, the expression of the PRC2binding domain of HOTAIR ( $5^{\prime}$ domain) but not of the LSD1-binding domain of HOTAIR ( $3^{\prime}$ domain) resulted in accelerated cell cycle progression. In conclusion, HOTAIR promotes cell cycle progression in glioma as a result of the binding of its $5^{\prime}$ domain to the PRC2 complex.

\section{INTRODUCTION}

Glioblastoma (GBM) represents the most aggressive and deadly primary brain tumor. The median survival of GBM patients is approximately 12 months from the time of diagnosis [1]. Patients benefit from maximal surgical 
resection, followed by radiotherapy and chemotherapy [2-3]. However, GBM cannot be fully resected because of its infiltrative growth. It is difficult to find a balance between the extent of resection and neurological morbidity. Even when maximal resection is achieved, tumor recurrence has been detected within the radiation field in most patients [4]. The cell cycle lies at the heart of cancer, and deregulated cell cycle progression can result in uncontrolled cancer cell proliferation. Temozolomide (TMZ) is an alkylating agent that is used in the treatment of GBM. TMZ therapy elicits anti-tumor activity by alkylating/methylating DNA at the N-7 or O-6 positions of guanine residues. As a result, TMZ induces DNA damage and death of tumor cells. Although TMZ has been used to treat newly diagnosed GBM since 2005 as a standardof-care treatment, the median survival time of all GBM patients after diagnosis remains less than 12 months [5]. These findings indicate that cell cycle progression is complicated in GBM. Thus, understanding the underlying mechanisms that regulate cell cycle progression in GBM might provide significant insight into the enhancement of the therapeutic management of GBM.

Non-coding RNAs (ncRNAs) are functional RNAs that do not encode proteins. NcRNAs include microRNAs (miRNAs), long non-coding RNAs (lncRNAs), tRNAs, snoRNAs, and siRNAs [6-7]. miRNAs have garnered significant attention over the past decade. Increasing studies have demonstrated that miRNAs are involved in the proliferation, invasion, apoptosis and cell cycle progression of cancer cells by binding to their mRNA targets [8-12]. Different from miRNAs, lncRNAs generally comprise non-protein-coding RNAs that consist of more than 200 nucleotides [13]. Recent reports have indicated that lncRNAs can serve as prognostic markers in various cancer types. Examples include HOTAIR in colorectal cancer and MALAT1 in non-small cell lung cancer [14-15]. HOTAIR and MALAT1 have also been demonstrated to be involved in breast and colorectal cancer metastasis [16-17]. Our previous studies showed that HOTAIR is overexpressed in high-grade glioma patients, and its upregulation is predictive of poor prognosis. In addition, gene set enrichment analysis has indicated that HOTAIR expression is involved in cell cycle progression [18]. However, the precise mechanism underlying how HOTAIR regulates cell cycle progression of glioma cells remains largely unknown. A study by Chang et al. demonstrated that HOTAIR serves as modular scaffold for two histone modification complexes. The 5' and 3' domains of HOTAIR bind to the PRC2 and LSD1 complexes, respectively [19]. The HOTAIR-mediated mechanism that regulates cell cycle progression via these two complexes in glioma cells remains unknown.

In this study, we aim to clarify the mechanism underlying HOTAIR-mediated regulation of cell cycle progression in glioma cells, as well as the function of PRC2 and LSD1 in this process. We first found that
EZH2 inhibition elicits effects that are consistent with those elicited by HOTAIR-targeted siRNA. We also found that the expression of the 5' domain of HOTAIR partially rescues the cell cycle arrest induced by HOTAIR knock-down in glioma cells. Thus, we demonstrate that in glioma cells, HOTAIR promotes cell cycle progression in an EZH2-dependent manner. These observations could further our understanding on the regulatory system of cell cycle progression in glioma cells.

\section{RESULTS}

\section{EZH2 inhibitor blocks GBM cell cycle progression}

Previous studies have shown that HOTAIR might serve as scaffold for LSD1 and PRC2 complexes [20-22]. We sought to determine which complex is more crucial for HOTAIR-regulated GBM cell cycle progression. To this end, we used small molecule inhibitors of both LSD1 and $\mathrm{EZH} 2$ (main component of PRC2 complex) in our subsequent studies. Consistent with other studies, we used 2-PCPA (LSD1 inhibitor) and DZNep (EZH2 inhibitor) at concentrations of 100 and $1 \mu \mathrm{M}$, respectively [23-24]. The GBM cell lines U87 and LN229 were treated with 2-PCPA or DZNep for 24, 48 and $72 \mathrm{~h}$. Western blot analysis revealed that LSD1 expression decreased after 2-PCPA treatment. However, expression of the cyclin-dependent kinase inhibitors p21 and p16 were only slightly changed. In addition, the G1/S-specific proteins Cyclin D1 and Cyclin E expression did not significantly change (Figure 1A). In contrast, in DZNep-treated GBM cells, p16 and p21 were both significantly upregulated at these time points, particularly at $48 \mathrm{~h}$. On the other hand, Cyclin D1 and Cyclin E expression levels were dramatically reduced in cells treated for $48 \mathrm{~h}$ (Figure 1C). As EZH2 was previously demonstrated to catalyze $\mathrm{H} 3 \mathrm{~K} 27$ methylation [25], we also examined the expression of H2K27Me3. The results showed that after DZNep treatment, H2K27Me3 levels decreased, whereas $\mathrm{H} 2 \mathrm{~K} 4 \mathrm{Me} 3$ levels remained unchanged (Figure 1C). In addition, altered cell cycle distribution at G1 phase was detected in DZNep-treated but not in 2-PCPA-treated, U87 and LN229 cells (Figure $1 \mathrm{~B}$ and $\mathrm{D})$. Thus we confirmed that EZH2 but not LSD1 was involved in the cell cycle progression in GBM cells. It was evidenced by the observation that cell cycle progression in GBM cells was significantly blocked after DZNep treatment for $48 \mathrm{~h}$.

\section{DZNep elicits similar cell cycle effects as si- HOTAIR in GBM cells}

Next, we compared the cell cycle effects of HOTAIR siRNA, 2-PCPA and DZNep in GBM cells. U87 and LN229 GBM cells were treated with si-HOTAIR, 2-PCPA 


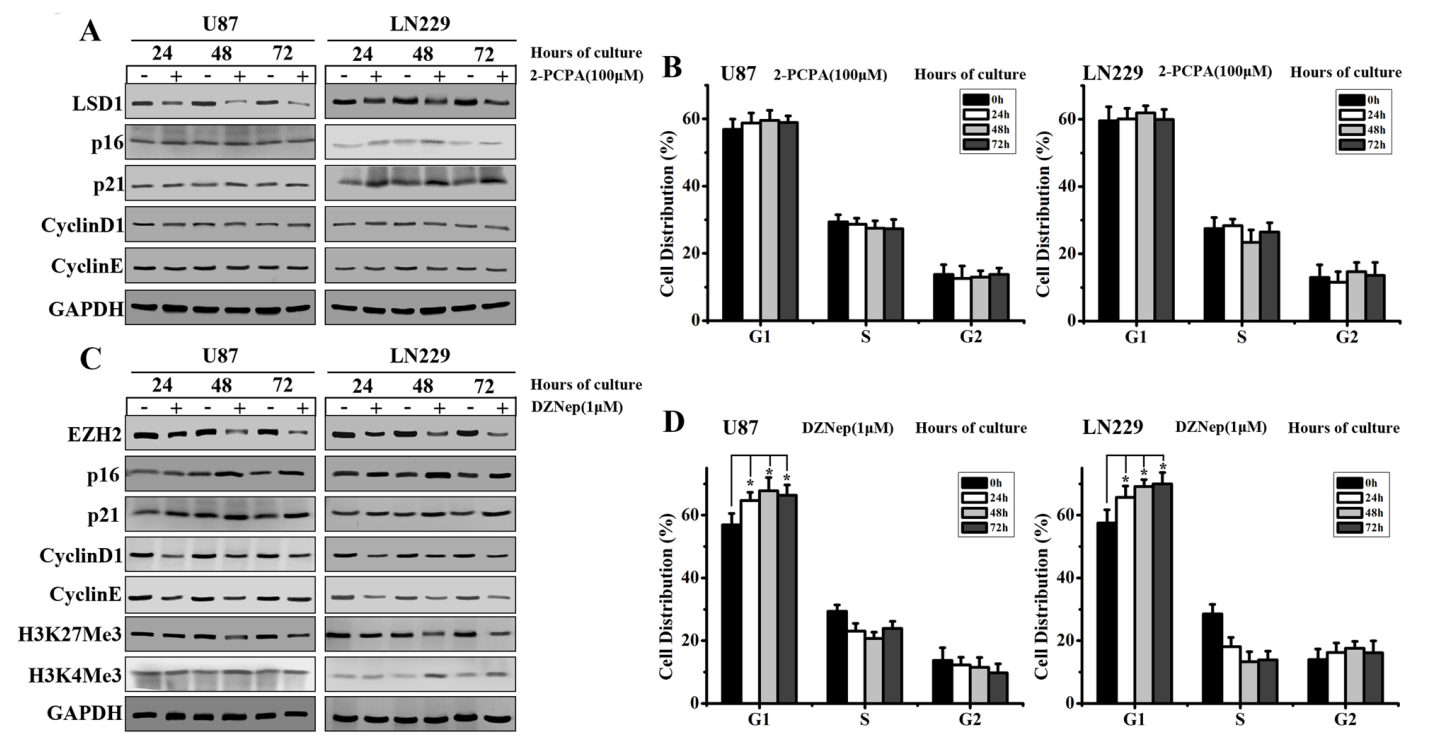

Figure 1: EZH2 inhibition blocks cell cycle progression in GBM cells. (A) Western blot analysis of U87 and LN229 cells shows the expression of LSD1, p16, p21, Cyclin D1 and Cyclin E after treatment with 2-PCPA (100 $\mu$ M) at 24, 48 and $72 \mathrm{~h}$, with GAPDH serving as a loading control. (B) Flow cytometry was performed to examine the G1/S arrest effect in U87 and LN229 cells after treatment with 2-PCPA $(100 \mu \mathrm{M})$ for 24, 48 and $72 \mathrm{~h}$. (C) Western blot analysis shows the expression of EZH2, p16, p21, Cyclin D1, Cyclin E, H3K27Me3 and H3K4Me3 in U87 and LN229 cells treated with DZNep $(1 \mu \mathrm{M})$ at 24, 48 and $72 \mathrm{~h}$, with GAPDH as a loading control. (D) Flow cytometry was performed to examine the G1/S arrest effect in U87 and LN229 after treatment with DZNep (1 $\mu$ M) for 24, 48 and $72 \mathrm{~h}$. The results presented represent mean values \pm SD of 3 independent experiments, which were each performed in triplicate. Student's paired $t$ tests were used to calculate $\mathrm{P}$ values, where $\mathrm{P}<0.05$ was considered to be statistically significant.
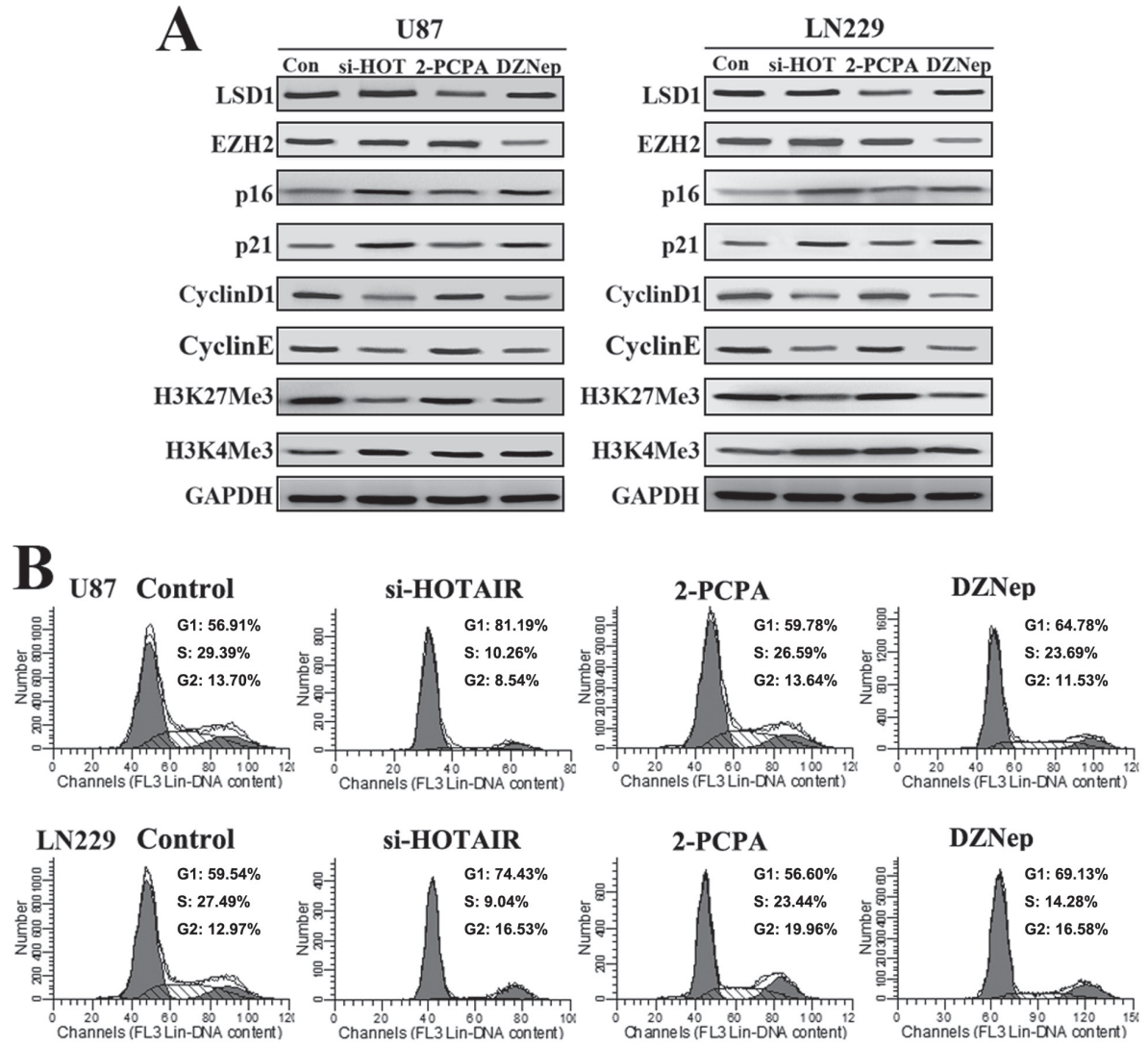

Figure 2: DZNep elicits similar cell cycle effects as si-HOTAIR in GBM cells. (A) Western blot analysis was used to examine the expression of LSD1, EZH2, p16, p21, Cyclin D1, Cyclin E, H3K27Me3 and H3K4Me3 in U87 and LN229 cells treated with siHOTAIR, 2-PCPA $(100 \mu \mathrm{M})$ and DZNep $(1 \mu \mathrm{M})$ for $48 \mathrm{~h}$. GAPDH was used as a loading control. (B) Flow cytometry was performed to

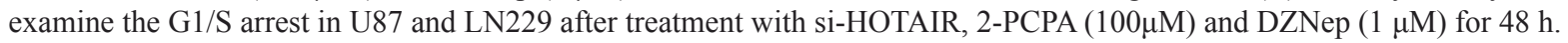


(100 $\mu \mathrm{M})$ and DZNep $(1 \mu \mathrm{M})$. Western blot analysis revealed that both $\mathrm{p} 16$ and $\mathrm{p} 21$ protein levels increased after si-HOTAIR and DZNep treatment compared with 2-PCPA. In addition, decreased Cyclin D1 and Cyclin E expression were detected after si-HOTAIR and DZNep treatment (Figure 2A). In addition, we detected that siHOTAIR inhibited H3K27Me3 to a similar extent as DZNep (Figure 2A). Next, flow cytometry was performed to examine cell cycle distribution. Our results revealed that DZNep elicited a similar G1/S cell cycle arrest as si-HOTAIR (Figure 2B). These data indicate that DZNep treatment elicits similar cell cycle effects as si-HOTAIR in GBM cells.

\section{HOTAIR inhibition suppresses tumor growth in a xenograft model predominantly via its 5 ' domain}

To evaluate the effects of si-HOTAIR, 2-PCPA and DZNep on tumor growth in vivo, we established intracranial xenograft tumors in nude mice. U87 cells were pretreated with a lentivirus containing a luciferase reporter. All of the mice generated tumors except for two mice within the si-HOTAIR treated group. Compared with 2-PCPA, both si-HOTAIR and DZNep treatments significantly decreased tumor burden (Figure 3A and B). At day 25 , one mouse was sacrificed in each group. HE staining revealed apoptotic morphological changes in si-HOTAIR- and DZNep-treated groups (Figure 3C). To analyze the survival times of the treatment groups, we then generated Kaplan-Meier survival curves, which demonstrated that si-HOTAIR and DZNep significantly prolonged survival (Figure 3D). These data showed that DZNep elicited similar effects as si-HOTAIR in regulating tumor growth in GBM xenograft model. Together with the findings in Figure 2, we propose that HOTAIR might modulate cell cycle progression in an EZH2-dependent manner in GBM cells.
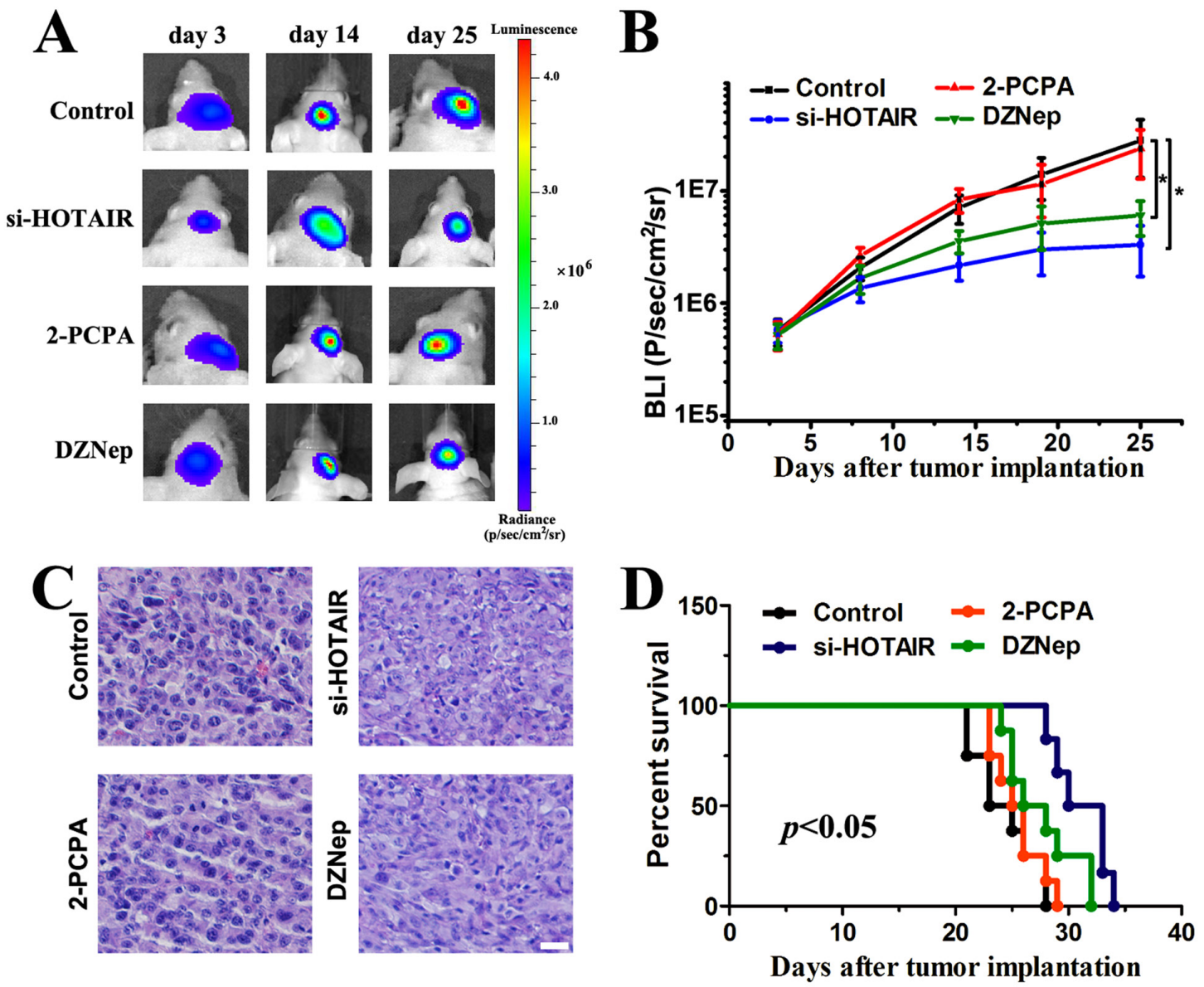

Figure 3: Si-HOTAIR and DZNep treatment prolongs survival in an intracranial glioma murine xenograft model. (A) U87 cells pretreated with a lentivirus containing luciferase were implanted in the right forebrain of nude mice, and tumor formation was assessed by bioluminescence imaging. Changes in bioluminescent signal were examined at day 3, 14 and 21 after tumor implantation. (B) The animals were intraperitoneally injected with 2-PCPA, DZNep or PBS (control) in each group, and bioluminescence was monitored to assess the tumor growth at day $3,8,14,19$ and $25 . * \mathrm{P}<0.05$. (C) At day 25 , one mouse was sacrificed in each group, and HE staining was used to examine morphological changes. (D) Overall survival was determined by Kaplan-Meier analysis, and log-rank test was used to assess the statistical significance of the differences. 


\section{Expression of the 5' domain of HOTAIR rescues the cell cycle progression in HOTAIR knock-down GBM cells}

To further confirm whether the regulation of cell cycle progression in GBM cells by HOTAIR is EZH2dependent, we infected U87 and LN229 cells with siHOTAIR lentivirus. A plasmid containing HOTAIR 3' and 5' domains were transfected at the same time. At $48 \mathrm{~h}$ after treatment, protein was prepared for western blot analysis, which revealed that si-HOTAIR blocked GBM cell cycle progress, as shown in Figure 2. After the expression of the HOTAIR 5' domain, the expression of p21, p16, Cyclin D1 and Cyclin E were almost restored to basal levels. However, the expression of the HOTAIR 3' domain did not change the expression of these proteins (Figure 4A). Next, flow cytometry was performed to examine cell cycle distribution. Our results showed that the expression of the HOTAIR 5' domain in si-HOTAIR-transfected cells rescued the G1/S cell cycle arrest (Figure 4B).

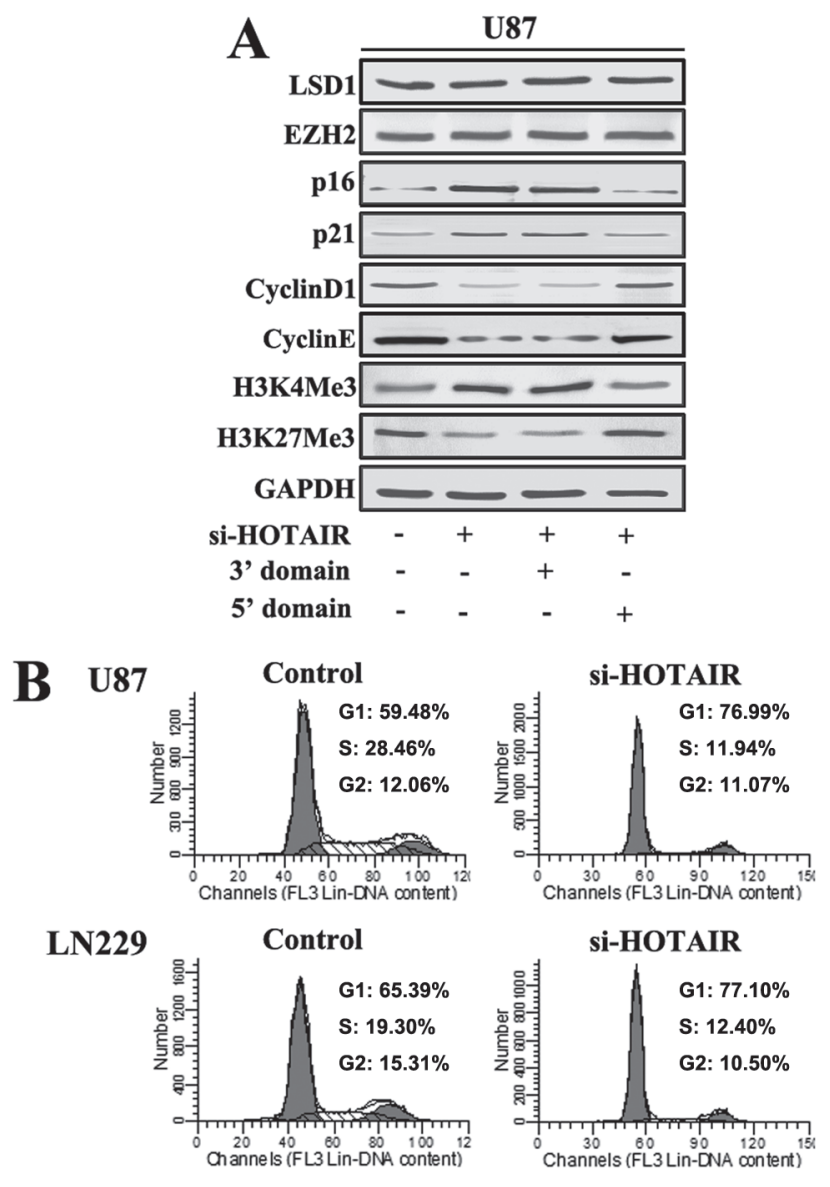

\section{Putative HOTAIR target genes are enriched for cell cycle regulatory function}

To identify putative HOTAIR target genes, we performed microarray analysis of 220 Chinese glioma samples. Gene ontology (GO) and KEGG analyses were used to annotate these target genes. GO analysis revealed that HOTAIR target genes are involved in cell cycle- and proliferation-related processes (with a $\mathrm{P}$ value of $1.86 \mathrm{E}$ 07) (Figure $5 \mathrm{~A}$ and Table 1). KEGG analysis revealed that 10 genes were enriched in cell cycle-related pathways (enrichment $\mathrm{P}$ value of 0.000155) (Figure 5B). Taken together, all these data demonstrate that si-HOTAIR inhibits GBM cell cycle progress in an EZH2-dependent manner.

\section{DISCUSSION}

HOTAIR has been extensively demonstrated to correlate with poor prognosis for breast, pancreatic and
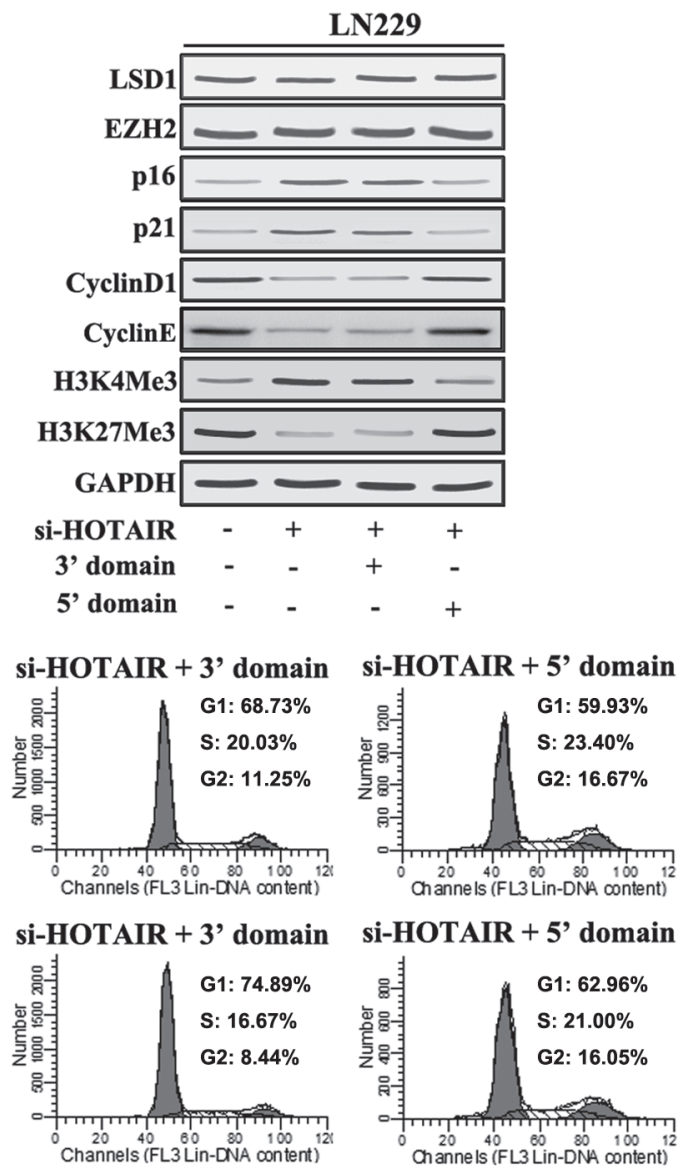

Figure 4: Expression of the 5' domain of HOTAIR rescues the cell cycle progression of HOTAIR knock-down GBM cells. U87 and LN229 GBM cells were infected with si-HOTAIR, and simultaneously transfected with either the 3' or 5' domains of HOTAIR. After $48 \mathrm{~h}$ of treatment, (A) Western blot analysis was performed on the expression of LSD1, EZH2, p16, p21, Cyclin D1, Cyclin E, H3K4Me3 and H3K27Me3; GAPDH was used as a loading control. (B) Flow cytometry was performed to examine the G1/S arrest effect. 
Table 1: P values of the Gene Ontology analysis results (genes with $\mathrm{P}<0.05$ are shown in red).

\begin{tabular}{|l|l|l|}
\hline term & count & P-value \\
\hline cell cycle and proliferation & 47 & $1.86 \mathrm{E}-07$ \\
\hline other metabolic processes & 47 & 0.593762 \\
\hline transport & 33 & 0.929507 \\
\hline stress response & 25 & 0.117135 \\
\hline developmental processes & 64 & 0.001425 \\
\hline RNA metabolism & 68 & 0.000403 \\
\hline DNA metabolism & 22 & $2.21 \mathrm{E}-06$ \\
\hline other biological processes & 106 & 0.88144 \\
\hline cell organization and biogenesis & 51 & 0.00072 \\
\hline cell-cell signaling & 9 & 0.120696 \\
\hline signal transduction & 36 & 0.999991 \\
\hline cell adhesion & 5 & 0.982058 \\
\hline protein metabolism & 50 & 0.313037 \\
\hline death & 13 & 0.663569 \\
\hline
\end{tabular}

colon cancer patients $[14,16,26]$. Furthermore, HOTAIR has been reported to regulate cancer cell cycle progression. However, whether HOTAIR is also involved in cell cycle regulation in glioma cells remains undetermined. This study identifies HOTAIR as a cell cycle-regulating lncRNA that is essential for glioma cell proliferation, indicating that HOTAIR might be a critical player in cell cycle progression in glioma cells.

Previous studies of HOTAIR have demonstrated that it can serve as a clinical prognostic marker. However, the precise regulatory mechanisms remain largely unknown. A study by Chang et al. previously showed that HOTAIR binds to PRC2 and LSD1 complexes via its 5' and 3' domains, respectively [19]. Gene set enrichment analysis based on cDNA microarray data from 32 colorectal cancer specimens showed that HOTAIR expression was significantly correlated with genome-wide retargeting of PRC2 genes [14]. Another microarray study of pancreatic cancer showed that GDF15 was regulated by both HOTAIR and PRC2. In contrast, interferon-related genes were not found to be affected by EZH2 knock-down but by HOTAIR-mediated suppression. These results indicated that HOTAIR-mediated gene repression in pancreatic cancer is both PRC2-dependent and PRC2-independent [26]. However, these studies focused on the HOTAIRPRC2 axis, while the HOTAIR-LSD1 axis remained unclear. In this study, we examined both HOTAIR-PRC2 and HOTAIR-LSD1 cell cycle regulatory functions in GBM. Our results suggest that in GBM cells, HOTAIR regulates cell cycle progression in an EZH2-dependent manner.

EZH2 is the catalytic subunit of PRC2, which
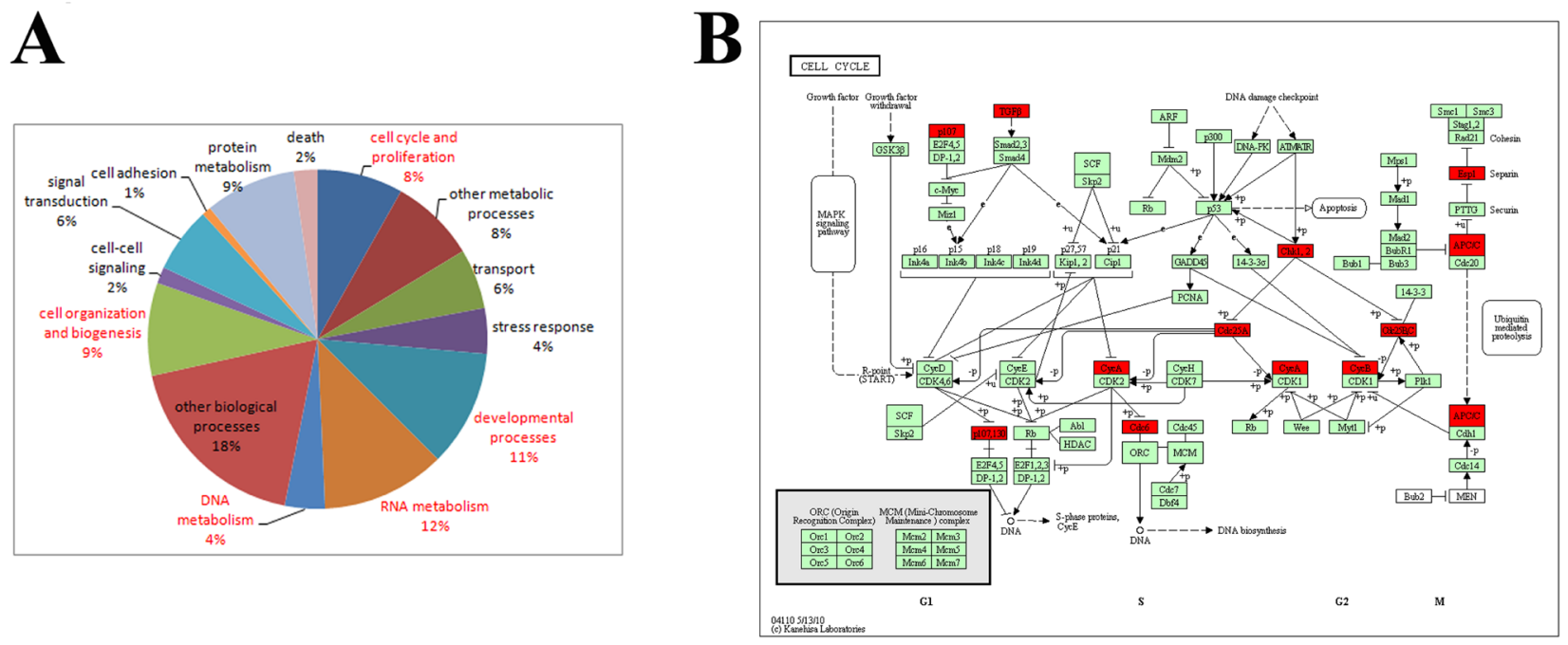

Figure 5: HOTAIR target genes are enriched for cell cycle regulatory genes. (A) Gene ontology results revealed that $8 \%$ of the target genes are involved in cell cycle and proliferation. The captions of GO terms in red indicate $\mathrm{P}$ value $<0.05$. (B) KEGG pathway analysis indicates that 10 of the target genes (enrichment $\mathrm{P}$ value 0.000155 ) represent key elements in cell cycle progression. 
functions as methyltransferase by adding three methyl groups to lysine 27 of histone 3 , a modification leading to chromatin condensation [27]. Previous studies have shown that EZH2 is overexpressed in glioma stem-like cells and adult glioblastoma patient samples [28-29]. The inhibition of EZH2 has been shown to induce cell cycle arrest at G0/ G1 phase in U87 human glioma cells [30]. In addition, EZH2 phosphorylation activates STAT3 signaling via STAT3 methylation and promotes the tumorigenicity of glioblastoma stem-like cells [31]. However, the function of LSD1 in human glioma remains largely unknown. A study by Singh et al. previously showed that LSD1 inhibition sensitized glioblastoma cells to histone deacetylase inhibitors [32]. In this study, we introduced small molecule inhibitor for both EZH2 and LSD1. GBM cells were treated with si-HOTAIR, an EZH2 inhibitor (DZNep) and a LSD1 inhibitor (2-PCPA). Our results demonstrate that DZNep elicits similar effects as those of si-HOTAIR in terms of glioma cell cycle distribution, whereas 2-PCPA only slightly altered cell cycle distribution. However, studies have shown that the proliferation of neuroblastoma and breast cancer cells was inhibited after treated with LSD1 inhibitor (TCP or TCP analogues) at concentrations 20-30-fold higher than enzymatic IC50 [33]. In HOTAIR knock-down glioma cells, unlike expression of the 5' domain, the expression of the HOTAIR 3' domain (LSD1 complex-binding domain) did not rescue the cell cycle arrest. These results demonstrate that in GBM cells, HOTAIR regulates cell cycle progression predominantly via the HOTAIR 5' domain-PRC2 axis, which is EZH2dependent.

In this study, we confirmed HOTAIR 5'domainPRC2 as a new regulatory axis that modulates cell cycle progression in GBM cells. A study by Li et al. showed that HOTAIR is overexpressed in laryngeal squamous cell carcinoma and regulates PTEN methylation [34]. A study by Lu et al. also showed that HOTAIR is involved in gene methylation in breast cancer [35]. However, these studies did not show why HOTAIR is relevant to methylation. As EZH2 is a methyltransferase, based on our findings in this study, we speculate that HOTAIR might also be related to gene methylation via the HOTAIR 5' domain-EZH2 axis.

Our in vivo study also showed that HOTAIR inhibition was therapeutically beneficial. The inhibition of HOTAIR slowed tumor growth and prolonged survival in a xenograft model. Our study suggests another lncRNAbased gene therapy approach for glioma patients. Because HOTAIR regulates cell cycle progression in GBM cells via its 5' domain, further examination and determination of the structure of HOTAIR, followed by molecular dockingbased virtual high-throughput screening techniques, might facilitate the discovery of small molecule inhibitors for HOTAIR.

Our present work uncovers a novel HOTAIRmediated mechanism of cell cycle regulation in GBM cells and provides a strong rationale for the further development of therapeutic strategies directly or indirectly targeting HOTAIR in GBM, by applying either DZNep or small molecule inhibitors for HOTAIR based on in silico 3D structural predictions.

\section{MATERIALS AND METHODS}

\section{Cell culture and drug treatment}

Human glioma cells (U87 and LN229) were obtained from ATCC (the American Type Culture Collection, Manassas, VA, USA) and were cultured in Dulbecco's modified Eagle medium (DMEM) supplemented with $10 \%$ heat-inactivated fetal bovine serum (FBS, Hyclone). The cells were maintained in a humidified atmosphere at $10 \% \mathrm{CO}_{2}$ atmosphere at $37^{\circ} \mathrm{C}$. The LSD1 inhibitor 2-PCPA (Sigma-Aldrich, USA) was dissolved in $\mathrm{H}_{2} \mathrm{O}$. The EZH2 inhibitor DZNep (Cayman, Michigan, USA) was dissolved in DMSO. The cells were treated with $100 \mu \mathrm{M}$ 2-PCPA or $1 \mu \mathrm{M}$ DZNep for 24,48 or $72 \mathrm{~h}$.

\section{Clinical samples and bioinformatics}

Two hundred and twenty glioma samples were collected from the Chinese Glioma Genome Atlas (CGGA, http://www.cgeg.org.cn/). As we previously described [36], there were 58 astrocytomas, 17 oligodendrogliomas, 22 oligoastrocytomas, 8 anaplastic astrocytomas, 11 anaplastic oligodendrogliomas, 15 anaplastic oligoastrocytomas, 4 secondary, and 85 primary GBMs. RNA was extracted from all of these tumor samples, and the Agilent Whole Human Genome Array was used for microarray analysis following the manufacturer's protocols. The GSEABase package from R (http://www.rproject.org/) statistical platform was used for Gene Ontology (GO) analysis. GO is to perform enrichment analysis on gene sets. GSEABase package provides classes and methods to support Gene Set Enrichment Analysis (GSEA). GSEA is a computational method that could be used to determine whether there is statistically significant difference on a defined set of genes between two biological states. In the set of genes that are upregulated by HOTAIR, an enrichment analysis will find which GO terms are over-represented by using annotations for that gene set. We also examined these genes in Matlab and mapped them to Kyoto Encyclopedia of Genes and Genomes (KEGG; http://www.genome.jp/kegg/ ) pathway database to analyze the correlation [37]. 


\section{Lentiviral infection and gene transfection}

Lentivirus containing HOTAIR siRNA segments (HOTAIR siRNA sequence is 5'-GAACGGGAGUACAGAGAGAUU-3') was obtained from Genepharma (Shanghai, China). U87 and LN229 cells were infected with the viral suspension. HOTAIR 3' domain (nucleotides 1 to 300 of HOTAIR) and 5' domain (nucleotides 1500 to 2146 of HOTAIR) were inserted into pcDNA3.1 $(+)$ plasmid. pcDNA3.1 (+)-3' domain and pcDNA3.1 (+)-5' domain plasmids were transfected using Lipofectamine 2000 (Invitrogen, Carlsbad, CA) following the manufacturer's protocols.

\section{Western blot}

Protein lysates were prepared as previously described [38]. The protein samples were resolved by SDS-PAGE and transferred onto PVDF membranes (Roche, Basel, Switzerland). The membranes were then incubated with the following antibodies: anti-LSD1 (Cell Signaling Technology), anti-EZH2 (Cell Signaling Technology), anti-p16 (Santa Cruz), anti-p21 (Santa Cruz), anti-Cyclin D1 (Santa Cruz), anti-Cyclin E (Santa Cruz), anti-H3K27Me3 (Millipore), and anti-H3K4Me3 (Millipore). Antibody-labeled protein bands on the PVDF membranes were detected using a G:BOX F3 (Syngene, Cambridge, UK).

\section{Cell cycle distribution}

U87 and LN229 cells $\left(1 \times 10^{5}\right.$ cells $)$ were plated in $60-\mathrm{mm}$ culture plates, and the cells were treated as previously described. After 2 days, the cells were trypsinized, fixed in $70 \%$ ethanol, washed once with PBS, and then labeled with propidium iodide (Sigma-Aldrich) in the presence of RNase A (Sigma-Aldrich) for $30 \mathrm{~min}$ in the dark $(50 \mathrm{~g} / \mathrm{mL})$. Samples were run on a FACScan flow cytometer (Becton-Dickinson, FL, NJ, USA), and the percentages of cells within each phase of the cell cycle were analyzed using Cell Quest software.

\section{Intracranial mice model}

All protocols involving animals were performed in accordance with an approved Institutional Animal Care and Use Committee protocol. Intracranial transplantation of GBM cells to establish GBM xenografts was performed as described previously [39-40]. Fifty thousand U87 cells (pretreated with either lentivirus containing with or without si-HOTAIR segments) were injected under the guidance of a stereotactic instrument. Three days after inoculation of the U87 cells (day 0), the treatments were initiated as follows: 2-PCPA $(2 \mathrm{mg} / \mathrm{kg})$ was injected intraperitoneally daily for 3 weeks, and DZNep was injected intraperitoneally $(2 \mathrm{mg} / \mathrm{kg})$ twice per week for 3 weeks. Bioluminescence imaging was used to detect intracranial tumor growth as previously described [40]. Identical circular regions of interest were drawn around the entire head of each animal to quantify bioluminescence. The Living Images software package (Caliper Life Sciences) was used to determine the integrated flux of photons (photons per second) within each region of interest. The data were normalized to the bioluminescence at the initiation of treatment for each animal. The error bars shown in the figures indicate the standard deviation (SD). A Kaplan-Meier survival curve is shown.

\section{HE staining}

The xenograft samples were collected at day 25 after tumor implantation, subjected to HE staining. For staining, $5-\mu \mathrm{m}$ sections were cut, dehydrated, deparaffinized, and rehydrated. HE staining was performed according to the standard protocols. All images were captured via microscopy (Olympus).

\section{ACKNOWLEDGMENTS}

This work was supported partially by the National High Technology Research and Development Program 863 (2014AA021102 and 2012AA02A508), the China National Natural Scientific Fund (81372703 and 81101916), the Natural Science Foundation of Tianjin Municipal Science and Technology Commission (12ZCDZSY17300), and the China Scholarship Council (CSC).

\section{Conflict of interest}

No potential conflicts of interest were disclosed.

\section{REFERENCES}

1. Stupp R, Mason WP, van den Bent MJ, Weller M, Fisher B, Taphoorn MJ, Belanger K, Brandes AA, Marosi C, Bogdahn U, Curschmann J, Janzer RC, Ludwin SK, Gorlia T, Allgeier A, Lacombe D, Cairncross JG, Eisenhauer E, Mirimanoff RO. Radiotherapy plus concomitant and adjuvant temozolomide for glioblastoma. N Engl J Med. 2005; 352:987-96.

2. Kuhnt D, Becker A, Ganslandt O, Bauer M, Buchfelder M, Nimsky C. Correlation of the extent of tumor volume resection and patient survival in surgery of glioblastoma multiforme with high-field intraoperative MRI guidance. Neuro Oncol. 2011; 13:1339-48.

3. Oppenlander ME, Wolf AB, Snyder LA, Bina R, Wilson JR, Coons SW, Ashby LS, Brachman D, Nakaji P, Porter 
RW, Smith KA, Spetzler RF, Sanai N. An extent of resection threshold for recurrent glioblastoma and its risk for neurological morbidity. J Neurosurg. 2014; 120:846-53.

4. Liang BC, Thornton AF, Jr., Sandler HM, Greenberg HS. Malignant astrocytomas: focal tumor recurrence after focal external beam radiation therapy. J Neurosurg. 1991; 75:55963.

5. Johnson DR, O'Neill BP. Glioblastoma survival in the United States before and during the temozolomide era. J Neurooncol. 2012; 107:359-64.

6. Mattick JS, Makunin IV. Non-coding RNA. Hum Mol Genet. 2006; 15 Spec No 1:R17-29.

7. Eddy SR. Non-coding RNA genes and the modern RNA world. Nature Reviews Genetics. 2001; 2:919-29.

8. Volinia S, Calin GA, Liu CG, Ambs S, Cimmino A, Petrocca F, Visone R, Iorio M, Roldo C, Ferracin M, Prueitt RL, Yanaihara N, Lanza G, Scarpa A, Vecchione A, Negrini M, Harris CC, Croce CM. A microRNA expression signature of human solid tumors defines cancer gene targets. Proc Natl Acad Sci U S A. 2006; 103:2257-61.

9. Hayashita Y, Osada H, Tatematsu Y, Yamada H, Yanagisawa K, Tomida S, Yatabe Y, Kawahara K, Sekido Y, Takahashi T. A polycistronic microRNA cluster, miR-17-92, is overexpressed in human lung cancers and enhances cell proliferation. Cancer Res. 2005; 65:9628-32.

10. Ma L, Teruya-Feldstein J, Weinberg RA. Tumour invasion and metastasis initiated by microRNA-10b in breast cancer. Nature. 2007; 449:682-8.

11. Li T, Li D, Sha J, Sun P, Huang Y. MicroRNA-21 directly targets MARCKS and promotes apoptosis resistance and invasion in prostate cancer cells. Biochem Biophys Res Commun. 2009; 383:280-5.

12. Petrocca F, Visone R, Onelli MR, Shah MH, Nicoloso MS, de Martino I, Iliopoulos D, Pilozzi E, Liu CG, Negrini M, Cavazzini L, Volinia S, Alder H, Ruco LP, Baldassarre G, Croce CM, Vecchione A. E2F1-regulated microRNAs impair TGFbeta-dependent cell-cycle arrest and apoptosis in gastric cancer. Cancer Cell. 2008; 13:272-86.

13. Mercer TR, Dinger ME, Mattick JS. Long non-coding RNAs: insights into functions. Nature Reviews Genetics. 2009; 10:155-9.

14. Kogo R, Shimamura T, Mimori K, Kawahara K, Imoto S, Sudo T, Tanaka F, Shibata K, Suzuki A, Komune S, Miyano S, Mori M. Long noncoding RNA HOTAIR regulates polycomb-dependent chromatin modification and is associated with poor prognosis in colorectal cancers. Cancer Res. 2011; 71:6320-6.

15. Schmidt LH, Spieker T, Koschmieder S, Schaffers S, Humberg J, Jungen D, Bulk E, Hascher A, Wittmer D, Marra A, Hillejan L, Wiebe K, Berdel WE, Wiewrodt R, Muller-Tidow C. The long noncoding MALAT-1 RNA indicates a poor prognosis in non-small cell lung cancer and induces migration and tumor growth. J Thorac Oncol. 2011; 6:1984-92.
16. Gupta RA, Shah N, Wang KC, Kim J, Horlings HM, Wong DJ, Tsai MC, Hung T, Argani P, Rinn JL, Wang Y, Brzoska P, Kong B, Li R, West RB, van de Vijver MJ, Sukumar S, Chang HY. Long non-coding RNA HOTAIR reprograms chromatin state to promote cancer metastasis. Nature. 2010; 464:1071-6.

17. Xu C, Yang M, Tian J, Wang X, Li Z. MALAT-1: a long non-coding RNA and its important 3' end functional motif in colorectal cancer metastasis. Int J Oncol. 2011; 39:16975.

18. Zhang JX, Han L, Bao ZS, Wang YY, Chen LY, Yan W, Yu SZ, Pu PY, Liu N, You YP, Jiang T, Kang CS. HOTAIR, a cell cycle-associated long noncoding RNA and a strong predictor of survival, is preferentially expressed in classical and mesenchymal glioma. Neuro Oncol. 2013; 15:1595603.

19. Tsai MC, Manor O, Wan Y, Mosammaparast N, Wang JK, Lan F, Shi Y, Segal E, Chang HY. Long noncoding RNA as modular scaffold of histone modification complexes. Science. 2010; 329:689-93.

20. Kaneko S, Li G, Son J, Xu CF, Margueron R, Neubert TA, Reinberg D. Phosphorylation of the PRC2 component Ezh2 is cell cycle-regulated and up-regulates its binding to ncRNA. Genes Dev. 2010; 24:2615-20.

21. Margueron R, Reinberg D. The Polycomb complex PRC2 and its mark in life. Nature. 2011; 469:343-9.

22. Margueron R, Li G, Sarma K, Blais A, Zavadil J, Woodcock CL, Dynlacht BD, Reinberg D. Ezh1 and Ezh2 maintain repressive chromatin through different mechanisms. Mol Cell. 2008; 32:503-18.

23. Schmidt DM, McCafferty DG. trans-2Phenylcyclopropylamine is a mechanism-based inactivator of the histone demethylase LSD1. Biochemistry. 2007; 46:4408-16.

24. Crea F, Hurt EM, Mathews LA, Cabarcas SM, Sun L, Marquez VE, Danesi R, Farrar WL. Pharmacologic disruption of Polycomb Repressive Complex 2 inhibits tumorigenicity and tumor progression in prostate cancer. Mol Cancer. 2011; 10:40.

25. Sarma K, Margueron R, Ivanov A, Pirrotta V, Reinberg D. Ezh2 requires PHF1 to efficiently catalyze H3 lysine 27 trimethylation in vivo. Mol Cell Biol. 2008; 28:2718-31.

26. Kim K, Jutooru I, Chadalapaka G, Johnson G, Frank J, Burghardt R, Kim S, Safe S. HOTAIR is a negative prognostic factor and exhibits pro-oncogenic activity in pancreatic cancer. Oncogene. 2013; 32:1616-25.

27. Varambally S, Dhanasekaran SM, Zhou M, Barrette TR, Kumar-Sinha C, Sanda MG, Ghosh D, Pienta KJ, Sewalt RG, Otte AP, Rubin MA, Chinnaiyan AM. The polycomb group protein EZH2 is involved in progression of prostate cancer. Nature. 2002; 419:624-9.

28. Orzan F, Pellegatta S, Poliani PL, Pisati F, Caldera V, Menghi F, Kapetis D, Marras C, Schiffer D, Finocchiaro G. Enhancer of Zeste 2 (EZH2) is up-regulated in malignant 
gliomas and in glioma stem-like cells. Neuropathol Appl Neurobiol. 2011; 37:381-94.

29. Suva ML, Riggi N, Janiszewska M, Radovanovic I, Provero P, Stehle JC, Baumer K, Le Bitoux MA, Marino D, Cironi L, Marquez VE, Clement V, Stamenkovic I. EZH2 is essential for glioblastoma cancer stem cell maintenance. Cancer Res. 2009; 69:9211-8.

30. Zhang R, Wang R, Chang H, Wu F, Liu C, Deng D, Fan W. Downregulation of Ezh2 expression by RNA interference induces cell cycle arrest in the G0/G1 phase and apoptosis in U87 human glioma cells. Oncol Rep. 2012; 28:2278-84.

31. Kim E, Kim M, Woo DH, Shin Y, Shin J, Chang N, Oh YT, Kim H, Rheey J, Nakano I, Lee C, Joo KM, Rich JN, Nam DH, Lee J. Phosphorylation of EZH2 activates STAT3 signaling via STAT3 methylation and promotes tumorigenicity of glioblastoma stem-like cells. Cancer Cell. 2013; 23:839-52.

32. Singh MM, Manton CA, Bhat KP, Tsai WW, Aldape K, Barton MC, Chandra J. Inhibition of LSD1 sensitizes glioblastoma cells to histone deacetylase inhibitors. Neuro Oncol. 2011; 13:894-903.

33. Lynch JT, Harris WJ, Somervaille TC. LSD1 inhibition: a therapeutic strategy in cancer? Expert Opin Ther Targets. 2012; 16:1239-49.

34. Li D, Feng J, Wu T, Wang Y, Sun Y, Ren J, Liu M. Long intergenic noncoding RNA HOTAIR is overexpressed and regulates PTEN methylation in laryngeal squamous cell carcinoma. Am J Pathol. 2013; 182:64-70.

35. Lu L, Zhu G, Zhang C, Deng Q, Katsaros D, Mayne ST, Risch HA, Mu L, Canuto EM, Gregori G, Benedetto C, $\mathrm{Yu} \mathrm{H}$. Association of large noncoding RNA HOTAIR expression and its downstream intergenic $\mathrm{CpG}$ island methylation with survival in breast cancer. Breast Cancer Res Treat. 2012; 136:875-83.

36. Zhang JX, Zhang J, Yan W, Wang YY, Han L, Yue X, Liu $\mathrm{N}$, You YP, Jiang T, Pu PY, Kang CS. Unique genomewide map of TCF4 and STAT3 targets using ChIP-seq reveals their association with new molecular subtypes of glioblastoma. Neuro Oncol. 2013; 15:279-89.

37. Bindea G, Mlecnik B, Hackl H, Charoentong P, Tosolini M, Kirilovsky A, Fridman WH, Pages F, Trajanoski Z, Galon J. ClueGO: a Cytoscape plug-in to decipher functionally grouped gene ontology and pathway annotation networks. Bioinformatics. 2009; 25:1091-3.

38. Chen L, Han L, Zhang K, Shi Z, Zhang J, Zhang A, Wang Y, Song Y, Li Y, Jiang T, Pu P, Jiang C, Kang C. VHL regulates the effects of miR-23b on glioma survival and invasion via suppression of HIF-1alpha/VEGF and betacatenin/Tcf-4 signaling. Neuro Oncol. 2012; 14:1026-36.

39. Zhang KL, Han L, Chen LY, Shi ZD, Yang M, Ren Y, Chen LC, Zhang JX, Pu PY, Kang CS. Blockage of a miR21/EGFR regulatory feedback loop augments anti-EGFR therapy in glioblastomas. Cancer Lett. 2014; 342:139-49.

40. Zhang KL, Zhou X, Han L, Chen LY, Chen LC, Shi ZD,
Yang M, Ren Y, Yang JX, Frank TS, Zhang CB, Zhang JX, $\mathrm{Pu}$ PY, Zhang JN, Jiang T, Wagner EJ, Li M, Kang CS. MicroRNA-566 activates EGFR signaling and its inhibition sensitizes glioblastoma cells to nimotuzumab. Mol Cancer. 2014; 13:63. 